\title{
Fuzzy Mappings and Fuzzy Equivalence Relations
}

\author{
Pyung Ki Lim ${ }^{1}$, Ga Hee $\mathrm{Choi}^{2}$ and Kul Hur ${ }^{3}$ \\ ${ }^{1,2,3}$ Division of Mathematics and Informational Statistics, and Nanoscale Science and \\ Technology Institute, Wonkwang University, Iksan, Chonbuk, Korea 570-749
}

\begin{abstract}
Equivalence relations and mappings for crisp sets are very well known. This paper attempts an investigation of equivalence relations and mappings for fuzzy sets. We list some concepts and results related to fuzzy relations. We give some examples corresponding to the concept of fuzzy equality and fuzzy mapping introduced by Demirci [1]. In addition, we introduce the notion of preimage and quotient of fuzzy equivalence relations. Finally, we investigate relations between a fuzzy equivalence relation and a fuzzy mapping.
\end{abstract}

Key Words : fuzzy mapping, fuzzy relation, fuzzy equivalence relation, fuzzy quotient of fuzzy mapping by fuzzy equivalence relation.

\section{Introduction}

The notion of fuzzy sets in a set generalises that of crisp subsets, and Zadeh[9] introduced it as an approach to a mathematical representation of vagueness in everyday language. Also a fuzzy relation between $X$ and $Y$ as a fuzzy set in $X \times Y$ was proposed by Zadeh[9]. Later he studied similarity relations in [10]. Subsequently, Goguen[2], Murali[3] and Ovchinnikov[5], etc., have studied fuzzy relations in various contents. Furthermore, Nemitz[4] have investigated fuzzy relations connected with equivalence relations and fuzzy functions. In particular, more recently, Demirci[1] studied fuzzy equalities and fuzzy mappings.

Equivalence relations and mappings in crisp set theory are very well known. This paper attempts an investigation of equivalence relations and mappings in fuzzy set theory. In Section 2, we list some concepts and results related to fuzzy relations. In Section 3 , we give some examples corresponding to the concept of fuzzy equality and fuzzy mapping introduced by Demirci[1]. Also, adding to his results, we obtain some another results. In Section 4, we introduce the notions of preimage and quotient of fuzzy equivalence relations. And we study some properties. In Section 5 , we investigate relations between a fuzzy equivalence

Manuscript received Jul. 12, 2011; revised Sep. 9, 2011; accepted Sep. 21, 2011.

${ }^{3}$ Corresponding author

This paper was supported by Wonkwang University in 2011. relation and a fuzzy mapping.

Throughout this paper, we denote the unit interval $[0,1]$ as $I$, and $X, Y, Z$, etc., denote ordinary sets. In particular, $I^{X}$ denotes the set of all fuzzy sets in $X$.

\section{Preliminaries}

In this section, we list some basic notions and results which are needed in the later sections.

Definition 2.1 [7]. Let $f: X \rightarrow Y$ be an (ordinary) mapping, let $A \in I^{X}$ and let $B \in I^{Y}$. Then:

(i) The image of $A$ under $f$, denoted by $f(A)$, is a fuzzy set in $Y$ defined as follows : For each $y \in Y$,

$$
[f(A)](y)= \begin{cases}\bigvee_{x \in f^{-1}(y)} A(x) & \text { if } f^{-1}(y) \neq \emptyset \\ 0 & \text { otherwise }\end{cases}
$$

(ii) The preimage of $B$ under $f$, denoted by $f^{-1}(B)$, is a fuzzy set in $X$ defined as follows : For each $x \in X$, $\left[f^{-1}(B)\right](x)=(B \circ f)(x)=B(f(x))$.

Result 2.A [8]. Let $f: X \rightarrow Y$ be an(ordinary) mapping, let $A \in I^{X}$ and $\left\{A_{\alpha}\right\}_{\alpha \in \Gamma} \subset I^{X}$, and let $B \in I^{Y}$ and $\left\{B_{\alpha}\right\}_{\alpha \in \Gamma} \subset I^{Y}$. Then: 
(a) $[f(A)]^{c} \subset f\left(A^{c}\right)$. In particular, $f$ is bijective, then $[f(A)]^{c}=f\left(A^{c}\right)$.

(b) $f^{-1}\left(B^{c}\right)=\left[f^{-1}(B)\right]^{c}$.

(c) If $A_{\alpha} \subset A_{\beta}$, for $\alpha, \beta \in \Gamma$, then $f\left(A_{\alpha}\right) \subset f\left(A_{\beta}\right)$.

(d) If $B_{\alpha} \subset B_{\beta}$, for $\alpha, \beta \in \Gamma$, then $f^{-1}\left(B_{\alpha}\right) \subset$ $f^{-1}\left(B_{\beta}\right)$.

(e) $A \subset f^{-1}(f(A))$. In particular, if $f$ is injective, then $f^{-1}(f(A))=A$.

(f) $f^{-1}(f(B)) \subset B$. In particular, if $f$ is surjective, then $f\left(f^{-1}(B)\right)=B$.

(g) $f\left(\bigcup_{\alpha \in \Gamma} A_{\alpha}\right)=\bigcup_{\alpha \in \Gamma} f\left(A_{\alpha}\right)$.

(h) $f^{-1}\left(\bigcup_{\alpha \in \Gamma} B_{\alpha}\right)=\bigcup_{\alpha \in \Gamma} f^{-1}\left(B_{\alpha}\right)$.

(i) $f\left(\bigcap_{\alpha \in \Gamma} A_{\alpha}\right) \subset \bigcap_{\alpha \in \Gamma} f\left(A_{\alpha}\right)$.

(j) $f^{-1}\left(\bigcap_{\alpha \in \Gamma} B_{\alpha}\right)=\bigcap_{\alpha \in \Gamma} f^{-1}\left(B_{\alpha}\right)$.

(h) If $g: Y \rightarrow Z$ is a mapping and $C \in I^{Z}$, then $(g \circ f)^{-1}(C)=f^{-1}\left(g^{-1}(C)\right)$, and $(g \circ f)(A)=g(f(A))$.

Definition 2.2 [9]. $R$ is called a fuzzy relation from $X$ to $Y$ (or a fuzzy relation on $X \times Y$ ) if $R \in I^{X \times Y}$, i.e, $R$ is a fuzzy set in $X \times Y$. In particular, if $R \in I^{X \times X}$, then $R$ is called a fuzzy relation on (or in ) $X$.

We will denote the set of all fuzzy relations on $X$ as $\operatorname{FR}(\mathrm{X})$.

Definition 2.3 [10]. Let $R \in I^{X \times Y}$ and $S \in I^{Y \times Z}$. Then:

(i) The sup-min composition of $R$ and $S$, denoted by $S \circ R$, is a fuzzy relation on $X \times Z$ defined as follows: $\forall x \in X, \forall z \in Z$,

$$
(S \circ R)(x, z)=\bigvee_{y \in Y}[R(x, y) \wedge S(y, z)] \text {. }
$$

(ii) The inverse of $R$, denoted by $R^{-1}$, is a fuzzy relation on $Y \times X$ defined by $R^{-1}(y, x)=R(x, y)$, $\forall(x, y) \in X \times Y$.

Definition $2.4[5,10]$. Let $R \in \operatorname{FR}(\mathrm{X})$. Then $R$ is said to be :

(i) reflexive if $R(x, x)=1, \forall x \in X$.

(ii) symmetric if $R(x, y)=R(y, x), \forall x, y \in X$, i.e, $R=R^{-1}$.

(iii) transitive if $R \circ R \subset R$,

(iv) a fuzzy equivalence relation on $X$ if it satisfies (i),(ii) and (iii).

We will denote the set of all fuzzy equivalence relation on $X$ as $\operatorname{FER}(\mathrm{X})$.
Let $R$ be a fuzzy equivalence relation on $X$ and let $a \in X$. We defined the mapping $R a: X \rightarrow I$ as follows : $\forall x \in X, R a(x)=R(a, x)$.

Then clearly $R a \in I^{X}$. In this case, $R a$ is called a fuzzy equivalence class of $R$ containing $a \in X$. The set $\{R a: a \in X\}$ is called the fuzzy quotient set of $X$ by $R$ and denoted by $X / R$ (See[5]).

Result 2.B [5, Lemma 2, Corollary and Theorem 1]. Let $R$ be a fuzzy equivalence relation on $X$. Then

(a) $R a=R b$ if and only if $R(a, b)=1, \forall a, b \in X$.

(b) $R a \cap R b=\varnothing$ if and only if $R(a, b)=0$, $\forall a, b \in X$.

(c) $\bigcup_{a \in X} R a=\mathrm{X}$.

\section{Fuzzy mappings}

In this section, we list some concepts and their properties by Demirci [1]. And we give some examples and obtain some results.

Definition 3.1[1]. A mapping $E_{X}: X \times X \rightarrow I$ is called a fuzzy equality on $X$ if it satisfies the following conditions :

(e.1) $E_{X}(x, y)=1 \Leftrightarrow x=y, \forall x, y \in X$,

(e.2) $E_{X}(x, y)=E_{X}(y, x), \forall x, y \in X$,

(e.3) $E_{X}(x, y) \wedge E_{X}(y, z) \leq E_{X}(x, z), \forall x, y, z \in X$.

We will denote the set of all fuzzy equalities as $E(X)$.

Example 3.1. Let $X=\{$ ᄀ, ᄂ, ᄃ $\}$ and let $E_{X}$ : $X \times X \rightarrow I$ be the mapping defined as following matrix

\begin{tabular}{c|ccc}
$E_{X}$ & $\neg$ & ᄂ & ᄃ \\
\hline ᄀ & 1 & 0.3 & 0.3 \\
ᄂ & 0.3 & 1 & 0.8 \\
ᄃ & 0.3 & 0.8 & 1
\end{tabular}

Then we can easily see that $E_{X} \in E(X)$.

Definition 3.2[1]. Let $f$ be a fuzzy relation on $X \times Y$. Then $f$ is called a fuzzy mapping with respect to (in short, w.r.t.) $E_{X} \in E(X)$ and $E_{Y} \in E(Y)$, denoted by $f: X \rightarrow Y$, if it satisfies the following condition :

(f.1) $\forall x \in X, \exists y \in Y$, such that $f(x, y)>0$,

(f.2) $\forall x_{1}, x_{2} \in X, \forall y_{1}, y_{2} \in Y, f\left(x_{1}, y_{1}\right) \wedge$ $f\left(x_{2}, y_{2}\right) \wedge E_{X}\left(x_{1}, x_{2}\right) \leq E_{Y}\left(y_{1}, y_{2}\right)$

Example 3.2. Let $X$ and $E_{X} \in E(X)$ be same as in Example 3.1. Let $Y=\{a, b\}$ and $E_{Y}: Y \times Y \rightarrow I$ be 
the mapping defined as follows :

\begin{tabular}{c|cc}
$E_{Y}$ & $a$ & $b$ \\
\hline$a$ & 1 & 0.7 \\
$b$ & 0.7 & 1
\end{tabular}

Then it is easily seen that $E_{Y} \in E(Y)$. Now define the fuzzy relation $f$ on $X \times Y$ follows :

\begin{tabular}{c|cc}
$f$ & $a$ & $b$ \\
\hline ᄀ & 0.5 & 1 \\
ᄂ & 0.4 & 0.7 \\
ᄃ & 1 & 0
\end{tabular}

Then we can easily prove that $f: X \rightarrow Y$ is a fuzzy mapping w.r.t. $E_{X}$ and $E_{Y}$.

Definition 3.3[1]. Let $f: X \rightarrow Y$ be a fuzzy mapping w.r.t. $E_{X} \in E(X)$ and $E_{Y} \in E(Y)$. Then $f$ is said to be :

(i) strong if $\forall x \in X, \exists y \in Y$ such that $f(x, y)=1$,

(ii) surjective if $\forall y \in Y, \exists x \in X$ such that $f(x, y)>0$,

(iii) strong surjective if $\forall y \in Y, \exists x \in X$ such that $f(x, y)=1$,

(iv) injective if $f\left(x_{1}, y_{1}\right) \wedge f\left(x_{2}, y_{2}\right) \wedge E_{Y}\left(y_{1}, y_{2}\right) \leq$ $E_{X}\left(x_{1}, x_{2}\right), \forall x_{1}, x_{2} \in X, \forall y_{1}, y_{2} \in Y$,

(v) bijective if it is surjective and injective,

(vi) strong bijective if it is strong surjective and injective.

Example 3.3. (a) Let $X, Y, E_{X}, E_{Y}$ and $f$ be same as in Example 3.2. Then $f: X \rightarrow Y$ is not strong but strong surjective. Moreover, it can be easily seen that $f$ is not injective.

(b) Let $X, Y, E_{X}$ and $E_{Y}$ be same as in Example 3.2. Define the fuzzy relation $g$ on $X \times Y$ as follows :

\begin{tabular}{c|cc}
$g$ & $a$ & $b$ \\
\hline ᄀ & 0.5 & 1 \\
ᄂ & 1 & 0.7 \\
ᄃ & 1 & 0
\end{tabular}

Then we can easily see that $g$ is strong and strong surjective w.r.t. $E_{X}$ and $E_{Y}$. But $g$ is not injective.

(c) Let $X, Y, E_{X}$ and $E_{Y}$ be same as in Example 3.2. Define the fuzzy relation $h$ on $Y \times X$ as follows :

\begin{tabular}{c|ccc}
$h$ & ᄀ & ᄂ & ᄃ \\
\hline$a$ & 0.5 & 0.4 & 1 \\
$b$ & 1 & 0.7 & 0
\end{tabular}

Then $h(a, \neg) \wedge h(b$, ᄂ $) \wedge E_{Y}(a, b)=0.5 \not \leq 0.3=E_{X}$ $(\neg$, ᄂ $)$. Thus $h$ is not a fuzzy mapping w.r.t. $E_{Y}$ and $E_{X}$.

(d) Let $X, Y, E_{X}$ and $E_{Y}$ be same as in Example 3.2. Define the fuzzy relation $k$ on $Y \times X$ as follows:

\begin{tabular}{c|ccc}
$k$ & ᄀ & ᄂ & ᄃ \\
\hline$a$ & 1 & 0.3 & 0.2 \\
$b$ & 1 & 0 & 0.3
\end{tabular}

Then we can easily show that $k: Y \rightarrow X$ is strong and injective w.r.t. $E_{Y}$ and $E_{X}$.

Definition 3.4[1]. The identify fuzzy mapping on $X$, denoted by $I_{X}$, is the fuzzy relation on $X \times X$ defined by:

$I_{X}(x, y)= \begin{cases}1 & \text { if } x=y, \\ 0 & \text { otherwise, for any } x, y \in X .\end{cases}$

Remark 3.4. (a) $I_{X}: X \rightarrow X$ is strong bijective w.r.t. any fuzzy equality on $X$. Moreover $I_{X}$ itself is a fuzzy equality on $X$.

(b) An (ordinary) mapping $f: X \rightarrow Y$ is a strong fuzzy mapping w.r.t. $E_{X}=I_{X} \in E(X)$ and $E_{Y}=I_{Y} \in E(Y)$

(c) Let $f: X \rightarrow Y$ be an (ordinary) mapping. If $f$ is injective [resp surjective and bijective], then $f$ is injective [resp. strong surjective and strong bijective] w.r.t. $I_{X} \in E_{X}$ and $I_{Y} \in E(Y)$.

Result 3.A[1, Proposition 2.1]. Let $f: X \rightarrow Y$ and $g: Y \rightarrow Z$ be fuzzy mappings w.r.t. $E_{X} \in \mathrm{E}(\mathrm{X})$, $E_{Y} \in \mathrm{E}(\mathrm{Y})$ and $E_{Z} \in \mathrm{E}(\mathrm{Z})$. Then sup-min composition $g \circ f$ is a fuzzy mapping $g \circ f: X \rightarrow Z$ w.r.t. $E_{X}$ and $E_{Z}$

Proposition 3.5. Let $f: X \rightarrow Y$ and $g: Y \rightarrow Z$ be fuzzy mappings w.r.t. $E_{X} \in E(X), E_{Y} \in E(Y)$ and $E_{Z} \in E(Z)$. If $f$ and $g$ are strong [resp. injective, surjective, strong surjective, bijective and strong bijective], then so is $g \circ f$.

Proof. (i) Suppose $f$ and $g$ are strong and let $x \in X$. Since $f$ is strong, $\exists y_{0} \in Y$ such that $f\left(x, y_{0}\right)=1$. Since $g$ is strong, $\exists z_{0} \in Z$ such that $g\left(y_{0}, z_{0}\right)=1$. Thus

$$
(g \circ f)\left(x, z_{0}\right)=\bigvee_{y \in Y}\left[f(x, y] \wedge g\left(y, z_{0}\right)\right] \geq f\left(x, y_{0}\right) \wedge
$$

$g\left(y_{0}, z_{0}\right)=1$.

So $g \circ f$ is strong.

(ii) Suppose $f$ and $g$ are surjective and let $z \in Z$. Since $g$ is surjective, $\exists y_{0} \in Y$ such that $g\left(y_{0}, z\right)>0$. Since $f$ is surjective, $\exists x_{0} \in X$ such that $f\left(x_{0}, y_{0}\right)>0$. Thus

$$
(g \circ f)\left(x_{0}, z\right)=\bigvee_{y \in Y}\left[f\left(x_{0}, y\right) \wedge g(y, z)\right] \geq f\left(x_{0}, y_{0}\right) \wedge
$$

$g\left(y_{0}, z\right)>0$.

So $g \circ f$ is surjective.

(iii) Suppose $f$ and $g$ are strong surjective and let $z \in Z$. Since $g$ is strong surjective, $\exists y_{0} \in Y$ such that 
$g\left(y_{0}, z\right)=1$. Since $f$ is strong surjective, $\exists x_{0} \in X$ such that $f\left(x_{0}, y_{0}\right)=1$. Thus

$g\left(y_{0}, z\right)=1$.

$$
(g \circ f)\left(x_{0}, z\right)=\bigvee_{y \in Y}[f(x, y) \wedge g(y, z)] \geq f\left(x_{0}, y_{0}\right) \wedge
$$

So $g \circ f$ is strong surjective.

(iv) Suppose $f$ and $g$ are injective. Let $x_{1}, x_{2} \in X$, let $y_{1}, y_{2} \in Y$ and let $z_{1}, z_{2} \in Z$.

Since $f$ is injective,

$$
f\left(x_{1}, y_{1}\right) \wedge f\left(x_{2}, y_{2}\right) \wedge E_{Y}\left(y_{1}, y_{2}\right)
$$

$E_{X}\left(x_{1}, x_{2}\right)$.

Since $g$ is injective,

$E_{Y}\left(y_{1}, y_{2}\right)$.

$$
g\left(y_{1}, z_{1}\right) \wedge g\left(y_{2}, z_{2}\right) \wedge E_{Z}\left(z_{1}, z_{2}\right) \leq
$$

By (3.1) and (3.2),

$$
\left(f\left(x_{1}, y_{1}\right) \wedge g\left(y_{1}, z_{1}\right)\right) \wedge\left(f\left(x_{2}, y_{2}\right) \wedge g\left(y_{2}, z_{2}\right)\right) \wedge
$$

$E_{Z}\left(z_{1}, z_{2}\right) \leq E_{X}\left(x_{1}, x_{2}\right)$.

Thus

$$
\left(\bigvee_{y \in Y}\left[f\left(x_{1}, y\right) \wedge g\left(y, z_{1}\right)\right]\right) \wedge\left(\bigvee_{y \in Y} f\left(x_{2}, y\right) \wedge g\left(y, z_{2}\right)\right) \wedge
$$

$E_{Z}\left(z_{1}, z_{2}\right)$

$\leq E_{X}\left(x_{1}, x_{2}\right)$. So $(g \circ f)\left(x_{1}, z_{1}\right) \wedge(g \circ f)\left(x_{2}, z_{2}\right) \wedge$

$E_{Z}\left(z_{1}, z_{2}\right) \leq E_{X}\left(x_{1}, x_{2}\right)$.

Hence $g \circ f$ is injective.

The remaiders are obvious by (i), (ii), (iii) and (iv).

Proposition 3.6. Let $f: X \rightarrow Y$ and $g: Y \rightarrow Z$ be fuzzy mappings w.r.t. $E_{X} \in E(X), E_{Y} \in E(Y)$ and $E_{Z} \in E(Z)$.

(a) If $g \circ f$ is strong, then so is $f$.

(b) If $g \circ f$ is surjective[resp. strong surjective], then so is $g$.

Proof.(a) Suppose $g \circ f$ is strong and let $x \in X$. Then $\exists Z_{0} \in Z$ such that $(g \circ f)\left(x, z_{0}\right)=1$. Thus $(g \circ f)\left(x, z_{0}\right)=\bigvee_{y \in Y}\left[f(x, y) \wedge g\left(y, z_{0}\right)\right]=1$. So $\exists y_{0} \in Y$ such that $f\left(x, y_{0}\right) \wedge g\left(y_{0}, z_{0}\right)=1$. In particular $f\left(x, y_{0}\right)=1$. Hence $f$ is strong.

(b) Suppose $g \circ f$ is surjective and let $z \in Z$. Then $\exists x_{0} \in X$ such that $(g \circ f)\left(x_{0}, z\right)>0$.

Thus

$$
\bigvee_{y \in Y}\left[f\left(x_{0}, y\right) \wedge g(y, z)\right]>0
$$

So $\exists y_{0} \in Y$ such that $f\left(x_{0}, y_{0}\right) \wedge g\left(y_{0}, z\right)>0$. In Particular, $g\left(y_{0}, z\right)>0$. Hence $g$ is surjective.

Now suppose $g \circ f$ is strong surjective and let $z \in Z$. Then $\exists x_{0} \in X$ such that $(g \circ f)\left(x_{0}, z\right)=1$.

Thus

$$
\bigvee_{y \in Y}\left[f\left(x_{0}, y\right) \wedge g(y, z)\right]=1
$$

So $\exists y_{0} \in Y$ such that $f\left(x_{0}, y_{0}\right) \wedge g\left(y_{0}, z\right)=1$. In particular, $g\left(y_{0}, z\right)=1$. Hence $g$ is surjective.
Let $f: X \rightarrow Y$ and $g: Y \rightarrow Z$ be two ordinary mappings. Then it is well-known that if $g \circ f: X \rightarrow Z$ is injective, then so is $f$. However, in case which $f$ and $g$ are fuzzy mappings, the above statement does not hold.

Example 3.6. Let $X=\left\{x_{1}, x_{2}, x_{3}\right\}, Y=\left\{y_{1}, y_{2}\right\}$ and $Z=\left\{z_{1}, z_{2}\right\}$, let $E_{X}: X \times X \rightarrow I$ be the mapping defined by $E_{X}\left(x_{i}, x_{i}\right)=1(i=1,2,3), E_{X}\left(x_{1}, x_{2}\right)=$ $E_{X}\left(x_{1}, x_{3}\right)=E_{X}\left(x_{2}, x_{1}\right)=E_{X}\left(x_{3}, x_{1}\right)=0$ and $E_{X}\left(x_{2}, x_{3}\right)=E_{X}\left(x_{3}, x_{2}\right)=0.5$. Then clearly $E_{X} \in$ $E(X)$.

Now let $f: X \times Y \rightarrow I$ and $g: Y \times Z \rightarrow I$ be the mappings defined as follows, respectively :

$$
f\left(x_{1}, y_{1}\right)=f\left(x_{2}, y_{2}\right)=1, f\left(x_{3}, y_{2}\right)=0.8
$$

and

$$
g\left(y_{1}, z_{1}\right)=1, g\left(y_{2}, z_{2}\right)=0.2 .
$$

Then we can easily see that $f$ is a fuzzy mapping w.r.t. $E_{X}$ and $I_{Y}$, and $g$ is a fuzzy mapping w.r.t. $I_{Y}$ and $I_{Z}$. Furthermore, we can see that $g \circ f: X \rightarrow Z$ is a fuzzy injective mapping w.r.t. $E_{X}$ and $I_{Z}$. But $f$ is not injective.

Definition 3.7. Let $f: X \rightarrow Y$ be a fuzzy mapping w.r.t. $E_{X} \in E(X)$ and $E_{Y} \in E(Y)$. Then $f$ is said to be invertible if the fuzzy relation $f^{-1}$ on $Y \times X$ is a fuzzy mapping $f^{-1}: Y \rightarrow X$ w.r.t. $E_{Y}$ and $E_{X}$.

Lemma 3.8. Let $f: X \rightarrow Y$ be a fuzzy mapping w.r.t. $E_{X} \in E(X)$ and $E_{Y} \in E(Y)$. If $f$ is invertible, then $f$ is bijective.

Proof. Suppose $f$ is invertible and let $y \in Y$. Since $f^{-1}: Y \rightarrow X$ is a fuzzy mapping w.r.t. $E_{Y}$ and $E_{X}$, $\exists x_{0} \in X$ such that $f^{-1}\left(y, x_{0}\right)>0$. Then $f\left(x_{0}, y\right)>0$. Thus $f$ is surjective. Now let $x_{1}, x_{2} \in X$ and let $y_{1}, y_{2} \in Y$. Since $f^{-1}: Y \rightarrow X$ is a fuzzy mapping, $f^{-1}\left(y_{1}, x_{1}\right) \wedge f^{-1}\left(y_{2}, x_{2}\right) \wedge E_{Y}\left(y_{1}, y_{2}\right) \leq E_{X}\left(x_{1}, x_{2}\right)$. Then $f\left(x_{1}, y_{1}\right) \wedge f\left(x_{2}, y_{2}\right) \wedge E_{Y}\left(y_{1}, y_{2}\right) \leq E_{X}\left(x_{1}, x_{2}\right)$. So $f$ is injective. Hence $f$ is bijective.

Lemma 3.9. Let $f: X \rightarrow Y$ be a bijective fuzzy mapping w.r.t. $E_{X} \in E(X)$ and $E_{Y} \in E(Y)$. Then the fuzzy relation $f^{-1}$ on $Y \times X$ is a bijective fuzzy mapping $f^{-1}: Y \rightarrow X$ w.r.t. $E_{Y}$ and $E_{X}$.

Proof. Let $y \in Y$. Since $f$ is surjective, $\exists x_{0} \in X$ such that $f\left(x_{0}, y\right)>0$. Then $f^{-1}\left(y, x_{0}\right)>0$. Thus $f^{-1}$ satisfies the condition (f.1). Now let $y_{1}, y_{2} \in Y$ and let $x_{1}, x_{2} \in X$. Since $f$ is injective, $f\left(x_{1}, y_{1}\right) \wedge f\left(x_{2}, y_{2}\right) \wedge E_{Y}\left(y_{1}, y_{2}\right) \leq E_{Y}\left(x_{1}, x_{2}\right)$. Then $f^{-1}\left(y_{1}, x_{1}\right) \wedge f^{-1}\left(y_{2}, x_{2}\right) \wedge E_{Y}\left(y_{1}, y_{2}\right) \leq E_{X}\left(x_{1}, x_{2}\right)$. Thus $f^{-1}$ satisfies the condition (f.2). So $f^{-1}$ : $Y \rightarrow X$ is fuzzy mapping w.r.t. $E_{Y}$ and $E_{X}$. Let $x \in X$. Since $f$ is a fuzzy mapping, $\exists y_{0} \in Y$ 
such that $f\left(x, y_{0}\right)>0$. Then $f^{-1}\left(y_{0}, x\right)>0$. Thus $f^{-1}$ is surjective. Now let $y_{1}, y_{2} \in Y$ and let $x_{1}, x_{2} \in X$. Since $f$ is a fuzzy mapping, $f\left(x_{1}, y_{1}\right) \wedge f\left(x_{2}, y_{2}\right) \wedge E_{X}\left(x_{1}, x_{2}\right) \leq E_{Y}\left(y_{1}, y_{2}\right)$. Then $f^{-1}\left(y_{1}, x_{1}\right) \wedge f^{-1}\left(y_{2}, x_{2}\right) \wedge E_{X}\left(x_{1}, x_{2}\right) \leq E_{Y}\left(y_{1}, y_{2}\right)$. Thus $f^{-1}$ is injective. So $f^{-1}$ is bijective. This competes the proof.

The following shows us that $f: X \rightarrow Y$ is strong surjective but $f^{-1}: Y \rightarrow X$ is not strong surjective. Thus $f$ is strong bijective but $f^{-1}$ is not strong bijective.

Example 3.9. Let $X, Y$ and $E_{X}$ be the same as in Example 3.6. We define the mapping $f: X \times Y \rightarrow I$ as follows:

$$
f\left(x_{1}, y_{1}\right)=f\left(x_{2}, y_{2}\right)=1 \text { and } f\left(x_{3}, y_{2}\right)=0.5 \text {. }
$$

Then we can easily check that $f$ is strong surjective but $f^{-1}$ is not strong surjective. Moreover, $f$ is injective. So $f$ is strong bijective but $f^{-1}$ is not strong bijective.

The following is the immediate result of Lemmas 3.8 and 3.9 .

Theorem 3.10. [1, Proposition 2.2]. Let $f: X \rightarrow Y$ be a fuzzy mapping w.r.t. $E_{X} \in E(X)$ and $E_{Y} \in$ $E(Y)$. Then $f$ is invertible if and only if $f$ is bijective.

Result 3.B[1, Proposition 2.3]. If $f: X \rightarrow Y$ is strong and injective w.r.t. $E_{X}=I_{X} \in E(X)$ and $E_{Y} \in E(Y)$, the $f^{-1} \circ f=I_{X}$.

Lemma 3.11. Let $f: X \rightarrow Y$ be a fuzzy mapping w.r.t. $E_{X} \in E(X)$ and $E_{Y} \in E(Y)$. If $f$ is strong surjective and $E_{Y}=I_{Y}$, then $f \circ f^{-1}=I_{Y}$.

Proof. Let $y, y^{\prime} \in Y$. Then

$$
\begin{aligned}
& \left(f \circ f^{-1}\right)\left(y, y^{\prime}\right) \\
= & \bigvee_{x \in X}\left[f^{-1}(y, x) \wedge f\left(x, y^{\prime}\right)\right] \\
= & \bigvee_{x \in X}\left[f(x, y) \wedge f\left(x, y^{\prime}\right)\right] \\
= & \bigvee_{x \in X}\left[f(x, y) \wedge f\left(x, y^{\prime}\right) \wedge E_{X}(x, x)\right]\left[\because E_{X}(x, x)=1\right] \\
\leq & E_{Y}\left(y, y^{\prime}\right)[\because f \text { is a fuzzy mapping }] \\
= & I_{Y}\left(y, y^{\prime}\right) .\left[\because E_{Y}=I_{Y}\right]
\end{aligned}
$$

Thus $f \circ f^{-1} \subset I_{Y}$. Now let $y, y^{\prime} \in Y$. Then clearly $I_{Y}\left(y, y^{\prime}\right)=1$ or $I_{Y}\left(y, y^{\prime}\right)=0$. If $I_{Y}\left(y, y^{\prime}\right)=0$, then clearly $I_{Y}\left(y, y^{\prime}\right) \leq\left(f \circ f^{-1}\right)\left(y, y^{\prime}\right)$. Suppose $I_{Y}\left(y, y^{\prime}\right)=1$, i.e, $y=y^{\prime}$. Since $f$ is strong surjective, for $y \in Y, \exists x_{0} \in X$ such that $f\left(x_{0}, y\right)=1$. Thus

$$
\begin{aligned}
& \left(f \circ f^{-1}\right)\left(y, y^{\prime}\right)=\left(f \circ f^{-1}\right)(y, y) \\
= & \bigvee_{x \in X}\left[f^{-1}(y, x) \wedge f(x, y)\right]
\end{aligned}
$$

$$
=\bigvee_{x \in X} f(x, y)=1
$$

So, in either cases, $I_{Y} \subset f \circ f^{-1}$. Hence $f \circ f^{-1}=$ $I_{Y}$.

The following is the immediate result of Result 3.B and Lemma 3.11 .

Theorem 3.12. Let $f: X \rightarrow Y$ be a fuzzy mapping w.r.t. $E_{X} \in E(X)$ and $E_{Y} \in E(Y)$. If $f$ is strong and strong bijective, $E_{X}=I_{X}$ and $E_{Y}=I_{Y}$, then $f^{-1} \circ f=I_{X}$ and $f \circ f^{-1}=I_{Y}$.

Result 3.C[1, Proposition 2.4]. Let $f: X \rightarrow Y$ and $g: Y \rightarrow Z$ be bijective w.r.t. $E_{X} \in E(X), E_{Y} \in$ $E(Y)$ and $E_{Z} \in E(Z)$. Then $(g \circ f)^{-1}=f^{-1} \circ g^{-1}$ and the fuzzy relation $(g \circ f)^{-1}$ is a fuzzy mapping $(g \circ f)^{-1}: Z \rightarrow X$ w.r.t. $E_{Z}$ and $E_{X}$.

Definition 3.13. [1]. Let $f: X \rightarrow Y$ be a fuzzy mapping, let $A \in I^{X}$ and let $B \in I^{Y}$. Then:

(i) The image of $A$ under $f$, denoted by $f(\mathrm{~A})$, is a fuzzy set in $\mathrm{Y}$ defined as follows:

$$
f(A)(y)=\bigvee_{x \in X}[A(x) \wedge f(x, y)], \forall y \in Y \text {. }
$$

(ii) The preimage of $B$ under $f$, denoted by $f^{-1}(B)$, is a fuzzy set in $\mathrm{X}$ defined as follows:

$$
f^{-1}(B)(x)=\bigvee_{y \in Y}[B(y) \wedge f(x, y)], \forall x \in X .
$$

Remark 3.13. (a) If $f: X \rightarrow Y$ is an (ordinary) mapping, then it is clear that Definition 3.13 is identical with Definition 2.1

(b) If $f: X \rightarrow Y$ is strong surjective, then $f(A)(y)=\bigvee_{f(x, y)=1} A(x), \forall y \in Y$.

(c) If $f: X \rightarrow Y$ is strong, then $f^{-1}(B)(x)=$ $\bigvee B(y), \forall x \in X$.

$f(x, y)=1$

The following is the immediate result of Definition 3.13 .

Proposition 3.14. Let $f: X \rightarrow Y$ and $g: Y \rightarrow Z$ be fuzzy mappings w.r.t. $E_{X} \in E(X), E_{Y} \in E(Y)$ and $E_{Z} \in E(Z)$, let $A \in I^{X}$ and let $B \in I^{Z}$. Then :

(a) $(g \circ f)(A)=g(f(A))$.

(b) $(g \circ f)^{-1}(B)=f^{-1}\left(g^{-1}(B)\right)$.

Result 3.D[1, Proposition 2.5]. Let $f: X \rightarrow Y$ be a fuzzy mapping w.r.t. $E_{X} \in E(X)$ and $E_{Y} \in E(Y)$, let $A \in I^{X}$ and let $B \in I^{Y}$.

(a) If $f$ is strong, then $A \subset f^{-1}(f(A))$.

(b) If $E_{X}=I_{X}$ and $f$ is injective, then $f^{-1}(f(A)) \subset A$.

(c) If $f$ is strong surjective, then $B \subset f\left(f^{-1}(B)\right)$. 
(d) If $E_{Y}=I_{Y}$, then $f\left(f^{-1}(B)\right) \subset B$.

The following is the immediate result of Theorem 2.5 in [6] and Definition 3.13.

Proposition 3.15. Let $f: X \rightarrow Y$ be a fuzzy mapping w.r.t. $E_{X} \in E(X)$ and $E_{Y} \in E(Y)$.

(a) Define the (ordinary) relation $\bar{f}$ from $I^{X}$ to $I^{Y}$ as follows: $\bar{f}(A)=f(A), \forall A \in I^{X}$. Then $\bar{f}: I^{X} \rightarrow I^{Y}$ is an (ordinary) mapping.

(b) Define the (ordinary) relation $\overline{\bar{f}}$ from $I^{Y}$ to $I^{X}$ as follows: $\overline{\bar{f}}(B)=f^{-1}(B), \forall B \in I^{Y}$. Then $\overline{\bar{f}}: I^{Y} \rightarrow I^{X}$ is an ordinary mapping.

The followings are the immediate results of Result 3.D and Proposition 3.14.

Corollary 3.15. Let $f: X \rightarrow Y$ be strong surjective w.r.t. $E_{X} \in E(X)$ and $E_{Y}=I_{y} \in E(Y)$. Then $\bar{f} \circ \overline{\bar{f}} \circ \bar{f}=\bar{f}$

Proof. Let $A \in I^{X}$. Since $f$ is strong surjective, by Result 3.D(c), $f(A) \subset f\left(f^{-1}(f(A))\right)$. Since $E_{Y}=I_{Y}$, by Result 3.D(d), $f\left(f^{-1}(f(A))\right) \subset f(A)$. So $f\left(f^{-1}(f(A))\right)=f(A)$. Hence $\bar{f} \circ \overline{\bar{f}} \circ \bar{f}=\bar{f}$.

Proposition 3.16. Let $f: X \rightarrow Y$ be a fuzzy mapping w.r.t. $E_{X} \in E(X)$ and $E_{Y} \in E(Y)$.

(a) If $f$ is strong, injective and $E_{X}=I_{X}$, then $\overline{\bar{f}} \circ \bar{f}$ is bijective. Hence $\bar{f}$ is injective and $\overline{\bar{f}}$ is surjective.

(b) If $f$ is strong surjective and $E_{Y}=I_{Y}$, then $\bar{f} \circ \overline{\bar{f}}$ is bijective. Hence $\bar{f}$ is surjective and $\overline{\bar{f}}$ is injective.

(c) If $f$ is strong, strong bijective, $E_{X}=I_{X}$ and $E_{Y}=I_{Y}$, then $\bar{f}$ and $\overline{\bar{f}}$ are bijective.

Proof.(a) Clearly $\overline{\bar{f}} \circ \bar{f}: I^{X} \rightarrow I^{X}$ is a mapping. Suppose $(\overline{\bar{f}} \circ \bar{f})\left(A_{1}\right)=(\overline{\bar{f}} \circ \bar{f})\left(A_{2}\right) \forall A_{1}, A_{2} \in I^{X}$. Then $\overline{\bar{f}}\left(\bar{f}\left(A_{1}\right)\right)=\overline{\bar{f}}\left(\bar{f}\left(A_{2}\right)\right)$. Thus, by the definitions of $\overline{\bar{f}}$ and $\bar{f}, f^{-1}\left(f\left(A_{1}\right)\right)=f^{-1}\left(f\left(A_{2}\right)\right)$. By Result 3.D,

$A_{1}=A_{2}$. So $\overline{\bar{f}} \circ \bar{f}$ is injective. Let $A \in I^{X}$. Then clearly $f(A) \in I^{Y}$. Moreover, by Result 3.D, $f^{-1}(f(A))=A$. Thus $(\overline{\bar{f}} \circ \bar{f})(A)=A$. So $\overline{\bar{f}} \circ \bar{f}$ is surjective. Hence $\overline{\bar{f}} \circ \bar{f}$ is bijective.

(b) Clearly $\bar{f} \circ \overline{\bar{f}}: I^{Y} \rightarrow I^{X}$ is a mapping. Suppose $(\bar{f} \circ \overline{\bar{f}})\left(B_{1}\right)=(\bar{f} \circ \overline{\bar{f}})\left(B_{2}\right), \forall B_{1}, B_{2} \in I^{Y}$. Then $\left.\bar{f}\left(\overline{\bar{f}} B_{1}\right)\right)=\bar{f}\left(\overline{\bar{f}}\left(B_{2}\right)\right)$, i.e, $f\left(f^{-1}\left(B_{1}\right)\right)=f\left(f^{-1}\left(B_{2}\right)\right)$. By Result 3.D, $B_{1}=B_{2}$. Thus $\bar{f} \circ \overline{\bar{f}}$ is injective. Let $B \in I^{Y}$. Then clearly $f^{-1}(B) \in I^{X}$ and $f\left(f^{-1}(B)\right)=B$. Thus $(\bar{f} \circ \overline{\bar{f}})(B)=B$. So $\bar{f} \circ \overline{\bar{f}}$ is surjective. Hence $\bar{f} \circ \overline{\bar{f}}$ is bijective. (c) It is clear from (a) and (b).

Result 3.E[1, Proposition 2.6]. Let $f: X \rightarrow Y$ be a fuzzy mapping w.r.t. $E_{X} \in E(X)$ and $E_{Y} \in E(Y)$, let $A \in I^{X}$ and let $B \in I^{Y}$.

(a) If $E_{X}=I_{X}$ and $f$ is injective, then $f\left(A^{c}\right) \subset$ $[f(A)]^{c}$.

(b) If $f$ is strong surjective, then $[f(A)]^{c} \subset f\left(A^{c}\right)$.

(c) If $f$ is strong, then $\left[f^{-1}(B)\right]^{c} \subset f^{-1}\left(B^{c}\right)$.

(d) If $E_{Y}=I_{Y}$, then $f^{-1}\left(B^{c}\right) \subset\left[f^{-1}(B)^{c}\right]$.

Result 3.F[1, Proposition 2.7]. Let $f: X \rightarrow Y$ be a fuzzy mapping w.r.t. $E_{X} \in E(X)$ and $E_{Y} \in E(Y)$, let $\left\{A_{\alpha}\right\}_{\alpha \in \Gamma} \subset I^{Y}$ and let $\left\{B_{\alpha}\right\}_{\alpha \in \Gamma} \subset I^{Y}$.

(a) $f\left(\bigcup_{\alpha \in \Gamma} A_{\alpha}\right)=\bigcup_{\alpha \in \Gamma} f\left(A_{\alpha}\right)$.

(b) $f^{-1}\left(\bigcup_{\alpha \in \Gamma} B_{\alpha}\right)=\bigcup_{\alpha \in \Gamma} f^{-1}\left(B_{\alpha}\right)$.

(c) $f\left(\bigcap_{\alpha \in \Gamma} A_{\alpha}\right) \subset \bigcap_{\alpha \in \Gamma} f\left(A_{\alpha}\right)$.

(d) $f^{-1}\left(\bigcap_{\alpha \in \Gamma} B_{\alpha}\right) \subset \bigcap_{\alpha \in \Gamma} f^{-1}\left(B_{\alpha}\right)$.

(e) If $A_{\alpha} \subset A_{\beta}$ for $\alpha, \beta \in \Gamma$, then $f\left(A_{\alpha}\right) \subset f\left(A_{\beta}\right)$.

(f) If $B_{\alpha} \subset B_{\beta}$ for $\alpha, \beta \in \Gamma$, then $f^{-1}\left(B_{\alpha}\right) \subset$ $f^{-1}\left(B_{\beta}\right)$.

(g) If $\mathrm{f}$ is injective and $E_{X}=I_{X}$, then $\bigcap_{\alpha \in \Gamma} f\left(A_{\alpha}\right) \subset$ $f\left(\bigcap_{\alpha \in \Gamma}\left(A_{\alpha}\right)\right.$.

(h) If $E_{Y}=I_{Y}$, then $\bigcap_{\alpha \in \Gamma} f^{-1}\left(B_{\alpha}\right) \subset f^{-1}\left(\bigcap_{\alpha \in \Gamma} B_{\alpha}\right)$.

The following is the immediate result of Definition 3.1

Proposition 3.17. Let $\left\{X_{\alpha}\right\}_{\alpha \in \Gamma}$ be a family of sets and let $X=\prod_{\alpha \in \Gamma} X_{\alpha}$ be the product of $\left\{X_{\alpha}\right\}_{\alpha \in \Gamma}$. If $E_{X_{\alpha}}$ is a fuzzy equality on $X_{\alpha}$ for each $\alpha \in \Gamma$, then $E_{X}=\prod_{\alpha \in \Gamma} E_{X_{\alpha}}$ is a fuzzy equality on $X$, where $E_{X}: X \times X \rightarrow I$ is the mapping defined as follows : $\forall\left(x_{\alpha}\right),\left(y_{\alpha}\right) \in X, E_{X}\left(\left(x_{\alpha}\right),\left(y_{\alpha}\right)\right)=\bigwedge_{\alpha \in \Gamma} E_{X_{\alpha}}\left(x_{\alpha}, y_{\alpha}\right)$.

The following is the immediate result of Definition 3.2 and Proposition 3.17.

Proposition 3.18. Let $X=\prod_{\alpha \in \Gamma} X_{\alpha}$ be the product of a family $\left\{X_{\alpha}\right\}_{\alpha \in \Gamma}$ of sets. For each $\alpha \in \Gamma$, we define the fuzzy relation $\pi_{\alpha}$ on $X \times X_{\alpha}$ as follows:

$\pi_{\alpha}\left(\left(x_{\alpha}\right), x\right)=\left\{\begin{array}{lll}1 & \text { if } \quad x=x_{\alpha}, \\ \geq 0 & \text { if } \quad x \neq x_{\alpha}, \forall\left(x_{\alpha}\right) \in X, \forall x \in X_{\alpha} .\end{array}\right.$ 
Then $\pi_{\alpha}: X \rightarrow X_{\alpha}$ is a fuzzy mapping w.r.t. $E_{X}=\prod_{\alpha \in \Gamma} E_{X_{\alpha}} \in E(X)$ and $E_{X_{\alpha}} \in E\left(X_{\alpha}\right), \forall \alpha \in \Gamma$. In this case, $\pi_{\alpha}$ is called the fuzzy projection of $X$ to $X_{\alpha}$.

From Proposition 3.18, it is clear that $\pi_{\alpha}$ is strong and strong surjective.

Proposition 3.19. Let $\pi_{\alpha}: X=\Pi_{\alpha \in \Gamma} X_{\alpha} \rightarrow X_{\alpha}$ be the fuzzy projection of $X$ to $X_{\alpha}$ and let $B_{\alpha} \in$ $I^{X_{\alpha}}, \forall \alpha \in \Gamma$. Then

$$
\bigcap_{\alpha \in \Gamma} \pi_{\alpha}^{-1}\left(B_{\alpha}\right)=\prod_{\alpha \in \Gamma} B_{\alpha}
$$

where $\prod_{\alpha \in \Gamma} B_{\alpha}$ is the fuzzy set in $X$ defined as follows:

$$
\left(\prod_{\alpha \in \Gamma} B_{\alpha}\right)\left(\left(x_{\alpha}\right)\right)=\bigwedge_{\alpha \in \Gamma} B_{\alpha}\left(x_{\alpha}\right), \forall\left(x_{\alpha}\right) \in X .
$$

Proof. Let $\left(x_{\alpha}\right) \in X$. Then

$$
\begin{aligned}
& {\left[\bigcap_{\alpha \in \Gamma} \pi_{\alpha}^{-1}\left(B_{\alpha}\right)\right]\left(\left(x_{\alpha}\right)\right) } \\
= & \bigwedge_{\alpha \in \Gamma} \pi_{\alpha}^{-1}\left(B_{\alpha}\right)\left(\left(x_{\alpha}\right)\right) \\
= & \bigwedge_{\alpha \in \Gamma} \bigvee_{x \in X_{\alpha}}\left[B_{\alpha}(x) \wedge \pi_{\alpha}\left(\left(x_{\alpha}\right), x\right)\right] \\
= & \bigwedge_{\alpha \in \Gamma} B_{\alpha}\left(x_{\alpha}\right)\left[\text { Since } \pi_{\alpha} \text { is strong }\right] \\
= & \left(\prod_{\alpha \in \Gamma} B_{\alpha}\right)\left(\left(x_{\alpha}\right)\right) .
\end{aligned}
$$

The following is the immediate result of Definition 3.2 and Proposition 3.17.

Proposition 3.20. Let $f: X \rightarrow Y$ be a fuzzy mapping w.r.t. $E_{X} \in E(X)$ and $E_{Y} \in E(Y)$. We define the fuzzy relation $g$ on $(X \times X) \times(Y \times Y)$ as follows : $g\left(\left(x, x^{\prime}\right),\left(y, y^{\prime}\right)=f(x, y) \wedge f\left(x^{\prime}, y^{\prime}\right), \forall\left(x, x^{\prime}\right) \in\right.$ $X \times X, \forall\left(y, y^{\prime}\right) \in Y \times Y$.

Then $g: X \times X \rightarrow Y \times Y$ is a fuzzy mapping w.r.t. $E_{X \times X}=E_{X} \times E_{X} \in E(X \times X)$ and $E_{Y \times Y}=$ $E_{Y} \times E_{Y} \in E(Y \times Y)$. In this case, $g$ is called the fuzzy product mapping of $f$ and is denoted by $g=f \times f=f^{2}$.

\section{Preimage and quotient of fuzzy equivalence relations.}

Proposition 4.1 Let $f: X \rightarrow Y$ be a strong fuzzy mapping w.r.t. $E_{X} \in E(X)$ and $E_{Y} \in E(Y)$, and let $R$ be a fuzzy equivalence relation on $Y$. Then $f^{-2}(R)$ is a fuzzy equivalence relation on $X$. In this case,
$f^{-2}(R)$ is called the preimage of $R$ under $f$, where $f^{-2}=\left(f^{2}\right)^{-1}=(f \times f)^{-1}$.

Proof. It is clear that $f^{-2}(R)$ is a fuzzy relation on $X$.

(i) Let $x \in X$. Then

$$
=\bigvee_{\left(y, y^{\prime}\right) \in Y \times Y}^{f^{-2}(R)(x, x)}\left[R\left(y, y^{\prime}\right) \wedge(f \times f)\left((x, x),\left(y, y^{\prime}\right)\right)\right]
$$

[By Definition 3.13 and Notation $f^{2}=f \times f$ ]

$$
=\bigvee_{\left(y, y^{\prime}\right) \in Y \times Y}\left[R\left(y, y^{\prime}\right) \wedge\left(f(x, y) \wedge f\left(x, y^{\prime}\right)\right)\right]
$$

[By Proposition 3.19]

$\geq R\left(y_{0}, y_{0}\right)$

[Since $f$ is strong, $\exists y_{0} \in Y$ such that $f\left(x, y_{0}\right)=1$.] $=1$.

Thus $f^{-2}(R)$ is reflexive.

(ii) By the definition of $f^{-2}(R)$, it is clear that $f^{-2}(R)$ is symmetric.

(iii) Let $x, x^{\prime \prime} \in X$. Then

$$
\begin{aligned}
& {\left[f^{-2}(R) \circ f^{-2}(R)\right]\left(x, x^{\prime \prime}\right)} \\
& =\bigvee_{x^{\prime} \in X}\left[f^{-2}(R)\left(x, x^{\prime}\right) \wedge f^{-2}(R)\left(x^{\prime}, x^{\prime \prime}\right)\right]
\end{aligned}
$$$$
=\bigvee_{x^{\prime} \in X}\left\{\left(\bigvee_{\left(y, y^{\prime}\right) \in Y \times Y}\left[R\left(y, y^{\prime}\right) \wedge(f \times f)\left(\left(x, x^{\prime}\right),\left(y, y^{\prime}\right)\right)\right]\right)\right.
$$$$
\wedge\left(\bigvee\left[R\left(y^{\prime}, y^{\prime \prime}\right) \wedge(f \times f)\left(\left(x^{\prime}, x^{\prime \prime}\right),\left(y^{\prime}, y^{\prime \prime}\right)\right)\right]\right\}
$$
$\left(y^{\prime}, y^{\prime \prime}\right) \in Y \times Y$

$$
=\bigvee_{x^{\prime} \in X}\left\{\left(\bigvee_{\left.y, y^{\prime}\right) \in Y \times Y}\left[R\left(y, y^{\prime}\right) \wedge f(x, y) \wedge f\left(x^{\prime}, y^{\prime}\right)\right] \wedge\right.\right.
$$

$\left(\bigvee_{y^{\prime}, y^{\prime \prime} \in Y}\left[R\left(y^{\prime}, y^{\prime \prime}\right) \wedge f\left(x^{\prime}, y^{\prime}\right) \wedge f\left(x^{\prime \prime}, y^{\prime \prime}\right)\right]\right\}$

$$
=\bigvee_{\left(y, y^{\prime \prime}\right) \in Y \times Y}\left[R\left(y, y_{0}\right) \wedge R\left(y_{0}, y^{\prime \prime}\right) \wedge f(x, y) \wedge\right.
$$
$\left.f\left(x^{\prime \prime}, y^{\prime \prime}\right)\right]$

[Since $f$ is strong, $\exists y_{0} \in Y$ such that $f\left(x^{\prime}, y_{0}\right)=1$ ]

$$
\begin{aligned}
& \leq \bigvee_{\left(y, y^{\prime \prime}\right) \in Y \times Y}\left[R\left(y, y^{\prime \prime}\right) \wedge(f \times f)\left(\left(x, x^{\prime \prime}\right),\left(y, y^{\prime \prime}\right)\right)\right] \\
& {[\because R \text { is transitive }] .} \\
& =f^{-2}(R)\left(x, x^{\prime \prime}\right) .
\end{aligned}
$$

Thus $f^{-2}(R) \circ f^{-2}(R) \subset f^{-2}(R)$. So $f^{-2}(R)$ is transitive. Hence $f^{-2}(R)$ is fuzzy equivalence relation on $X$.

Corollary 4.1 Let $f$ and $R$ be same as in Proposition 4.1. Then $f^{-2}(R)=f^{-1} \circ R \circ f$.

Proof. Let $a, b \in X$. Then

$$
\begin{aligned}
f)((a, b),(c, d))] & f^{-2}(R)(a, b)=\bigvee_{(c, d) \in Y \times Y}[R(c, d) \wedge(f \times \\
= & \bigvee_{(c, d) \in Y \times Y}[R(c, d) \wedge f(a, c) \wedge f(b, d)] \\
= & \bigvee_{d \in Y}\left\{\left(\bigvee_{c \in Y}[f(a, c) \wedge R(c, d)]\right) \wedge f(b, d)\right\}
\end{aligned}
$$




$$
\begin{aligned}
& =\bigvee_{d \in Y}[(R \circ f)(a, d) \wedge f(b, d)] \\
& =\bigvee_{d \in Y}\left[(R \circ f)(a, d) \wedge f^{-1}(d, b)\right] \\
& =\left(f^{-1} \circ(R \circ f)\right)(a, b) .
\end{aligned}
$$

Hence $f^{-2}(R)=f^{-1} \circ R \circ f$.

Proposition 4.2 If $R$ is a fuzzy equivalence relation on $X$, then $\exists$ the strong and strong surjective fuzzy mapping $\pi: X \rightarrow X / R$ w.r.t. $I_{X} \in E(X)$ and $E_{X / R} \in E(X / R)$, where $E_{X / R}: X / R \times X / R \rightarrow I$ is the fuzzy equality on $X / R$ defined as follows : $\forall a, b \in X$,

$$
E_{X / R}(R a, R b)=R(a, b) \text {. }
$$

In this case, $\pi$ is called the natural (or canonical) fuzzy mapping.

Proof. We define the fuzzy relation $\pi: X \times X / R \rightarrow I$ as follows : $\forall a, b \in X$,

$$
\pi(a, R b)=R b(a)=R(b, a) .
$$

Then clearly $\pi$ satisfies the condition (f.1). Let $a_{1}, a_{2}, b_{1}, b_{2} \in X$. If $a_{1} \neq a_{2}$, then clearly $I_{X}\left(a_{1}, a_{2}\right)=$ 0 . Thus

$$
\pi\left(a_{1}, R b_{1}\right) \wedge \pi\left(a_{2}, R b_{2}\right) \wedge I_{X}\left(a_{1}, a_{2}\right) \leq
$$

$E_{X / R}\left(R b_{1}, R b_{2}\right)$.

Suppose $a_{1}=a_{2}$. Then

$$
\begin{aligned}
& \pi\left(a_{1}, R b_{1}\right) \wedge \pi\left(a_{2}, R b_{2}\right) \wedge I_{X}\left(a_{1}, a_{2}\right) \\
= & R\left(a_{1}, b_{1}\right) \wedge R\left(a_{1}, b_{2}\right) \wedge I_{X}\left(a_{1}, a_{1}\right) \\
= & R\left(b_{1}, a_{1}\right) \wedge R\left(a_{1}, b_{2}\right) \\
& {\left[\text { Since } R \text { is symmetric and } I_{X}\left(a_{1}, a_{1}\right)=1\right] . } \\
\leq & R\left(b_{1}, b_{2}\right)[\text { Since } R \text { is transitive]. } \\
= & E_{X / R}\left(R b_{1}, R b_{2}\right) .
\end{aligned}
$$

Thus $\pi$ satisfies the condition (f.2). So $\pi: X \rightarrow X / R$ is a fuzzy mapping w.r.t. $I_{X}$ and $E_{X / R}$. Moreover, it is clear that $\pi$ is strong and strong surjective from the definition of $\pi$.

Proposition 4.3 Let $R$ and $G$ be fuzzy equivalence relations on $X$ such that $R \subset G$. We define the mapping $G / R: X / R \times X / R \rightarrow I$ as follows :

$$
G / R(R a, R b)=G(a, b), \forall a, b \in X .
$$

Then $G / R$ is a fuzzy equivalence relation on $X / R$. In this case, $G / R$ is called the fuzzy quotient of $G$ by $R$.

Proof. It is clear that $G / R$ is reflexive and symmetric. Let $a, c \in X$. Then

$$
\begin{aligned}
& (G / R \circ G / R)(R a, R c) \\
= & \bigvee_{b \in X}[G / R(R a, R b) \wedge G / R(R b, R c)] \\
= & \bigvee_{b \in X}[G(a, b) \wedge G(b, c)] \\
= & (G \circ G)(a, c) \\
\leq & G(a, c)[\because G \text { is transitive }] \\
= & G / R(R a, R c) .
\end{aligned}
$$

Hence $G / R$ is a fuzzy equivalence relation on $X / R$.
The following is the immediate result of Proposition 4.3 .

Corollary 4.3 Let $R, G$ and $H$ be fuzzy equivalence relations on $X$ such that $R \subset G \subset H$. Then $G / R \subset H / R$.

Proposition 4.4 Let $R, G$ and $H$ be fuzzy equivalence relation on $X$ such that $R \subset G \subset H$.

(a) $R \subset G \circ H$.

(b) If $G \circ H$ is a fuzzy equivalence relation on $X$, then $(G \circ H) / R$ is a fuzzy equivalence relation on $X / R$ and $G / R \circ H / R=(G \circ H) / R$. $X / R$.

(c) $G / R \circ H / R$ is a fuzzy equivalence relation on

Proof. (a) Let $a, c \in X$. Then

$(G \circ H)(a, c)$

$=\bigvee_{b \in X}[H(a, b) \wedge G(b, c)]$

$\geq \bigvee_{b \in X}[R(a, b) \wedge R(b, c)][\because R \subset G \subset H]$

$\geq R(a, c) \wedge R(c, c)$

$=R(a, c) .[\because R(c, c)=1]$

Thus $R \subset G \circ H$.

(b) By the hypothesis and, (a) and Proposition 4.3, it is clear that $(G \circ H) / R$ is a fuzzy equivalence relation on $X / R$. Let $a, c \in X$. Then

$(G / R \circ H / R)(R a, R c)$

$=\bigvee_{b \in X}[H / R(R a, R b) \wedge G / R(R b, R c)]$

$=\bigvee_{b \in X}[H(a, b) \wedge G(b, c)]$

$=(G \circ H)(a, c)$

$=[(G \circ H) / R](R a, R c)$.

Thus $G / R \circ H / R=(G \circ H) / R$.

(c) It is obvious from (b).

Proposition 4.5 Let $R$ and $G$ be fuzzy equivalence relations on $X$ and $Y$, respestively . Let the fuzzy product of $R$ and $G$, denoted by $R \cdot G$, be a fuzzy relation on $(X \times Y) \times(X \times Y)$ defined as follows : $\forall x_{1}, x_{2} \in X, \forall y_{1}, y_{2} \in Y$

$(R \cdot G)\left(\left(x_{1}, y_{1}\right),\left(x_{2}, y_{2}\right)\right)=R\left(x_{1}, x_{2}\right) \wedge G\left(y_{1}, y_{2}\right)$. Then $R \cdot G$ is a fuzzy equivalence relation on $X \times Y$.

Proof. Let $(a, b) \in X \times Y$. Then

$$
(R \cdot G)((a, b),(a, b))
$$

$=R(a, a) \wedge G(b, b)$.

$=1$. $[\because R$ and $G$ are fuzzy equivalence relations $]$

Thus $R \cdot G$ is reflexive. It is clear that $R \cdot G$ is symmetric. Now let $\left(a_{1}, b_{1}\right),\left(a_{3}, b_{3}\right) \in X \times Y$. Then

$$
[(R \cdot G) \circ(R \cdot G)]\left(\left(a_{1} \cdot b_{1}\right),\left(a_{3}, b_{3}\right)\right)
$$




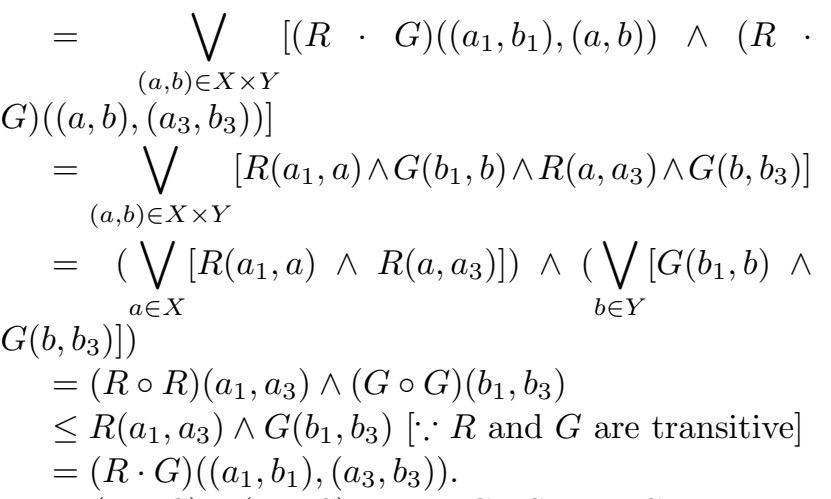

Thus $(R \cdot G) \circ(R \cdot G) \subset R \cdot G$. So $R \cdot G$ is transitive. Hence $R \cdot G$ is a fuzzy equivalence relation on $X \times Y$.

\section{Fuzzy equivalence relations and fuzzy mappings.}

Proposition 5.1 Let $f: X \rightarrow Y$ be a strong fuzzy mapping w.r.t. $E_{X} \in E(X)$ and $E_{Y} \in E(Y)$. We define the mapping $R: X \times X \rightarrow I$ as follows :

$$
R\left(x, x^{\prime}\right)=\bigvee_{\left(y, y^{\prime}\right) \in Y \times Y}\left[f(x, y) \wedge f\left(x^{\prime}, y^{\prime}\right) \wedge\right.
$$

$\left.E_{Y}\left(y, y^{\prime}\right)\right], \forall\left(x, x^{\prime}\right) \in X \times X$.

Then $R$ is a fuzzy equivalence relation on $X$. In this case, $R$ is called the fuzzy equivalence relation on $X$ determined by $f$ and will be denoted by $R_{f}$.

Proof. Let $a \in X$. Then

$$
\begin{aligned}
& \bigvee_{\left(b, b^{\prime}\right) \in Y \times Y}^{R(a, a)}\left[f(a, b) \wedge f\left(a, b^{\prime}\right) \wedge E_{Y}\left(b, b^{\prime}\right)\right] \\
\geq & f\left(a, b_{0}\right) \wedge f\left(a, b_{0}\right) \wedge E_{Y}\left(b_{0}, b_{0}\right)
\end{aligned}
$$

[Since $f$ is strong, $\exists b_{0} \in Y$ such that $f\left(a, b_{0}\right)=1$ ]

$$
=1 \text {. }
$$

Thus $R$ is reflexive. It is clear that $R$ is symmetric. Now let $a, c \in X$. Then

$$
\begin{aligned}
& (R \circ R)(a, c) \\
= & \bigvee_{x \in X}[R(a, x) \wedge R(x, c)] \\
= & \bigvee_{x \in X}\left\{\left(\bigvee_{\left(b, b^{\prime}\right) \in Y \times Y}\left[f(a, b) \wedge f\left(x, b^{\prime}\right) \wedge E_{Y}\left(b, b^{\prime}\right)\right]\right)\right. \\
\wedge & \left.\left(\bigvee_{\left(b^{\prime}, b^{\prime \prime}\right) \in Y \times Y}\left[f\left(x, b^{\prime}\right) \wedge f\left(c, b^{\prime \prime}\right) \wedge E_{Y}\left(b^{\prime}, b^{\prime \prime}\right)\right]\right)\right\} \\
= & \left(\bigvee_{\left(b, b_{0}\right) \in Y \times Y}\left[f(a, b) \wedge f\left(x, b_{0}\right) \wedge E_{Y}\left(b, b_{0}\right)\right]\right) \\
\wedge & \left(\bigvee_{b_{0}, b^{\prime \prime} \in Y \times Y}\left[f\left(x, b_{0}\right) \wedge f\left(c, b^{\prime \prime}\right) \wedge E_{Y}\left(b_{0}, b^{\prime \prime}\right)\right]\right)
\end{aligned}
$$

[Since $f$ is strong, $\exists b_{0} \in Y$ such that $f\left(x, b_{0}\right)=1$.]

$$
\begin{aligned}
& \leq \bigvee_{\left(b, b^{\prime \prime}\right) \in Y \times Y}\left[f(a, b) \wedge f\left(c, b^{\prime \prime}\right) \wedge E_{Y}\left(b, b^{\prime \prime}\right)\right] \\
& {\left[\because E_{Y} \text { is a fuzzy equality on } Y\right]} \\
& =R(a, c) .
\end{aligned}
$$

So $R$ is transitive. Hence $R$ is fuzzy equivalence relation on $X$.

The following is the immediate result of Propositions 4.2 and 5.1 .

Corollary 5.1 Let $R$ be a fuzzy equivalence relation on $X$. If $\pi: X \rightarrow X / R$ is the natural fuzzy mapping w.r.t. $I_{X} \in E(X)$ and $E_{X / R} \in E(X / R)$, then $R=R_{\pi}$.

Proof. From Proposition 4.2, it is clear that $\pi$ is strong and strong surjective. Let $a, b \in X$, Then

$$
\begin{aligned}
= & \bigvee_{(c, d) \in X \times X}\left[\pi(a, R c) \wedge \pi(b, R d) \wedge E_{X / R}(R c, R d)\right] \\
= & \bigvee_{(c, d) \in X \times X}[R(c, a) \wedge R(d, b) \wedge R(c, d)]
\end{aligned}
$$$$
\text { [By the definitions of } \pi \text { and } E_{X / R} \text {.] }
$$$$
=\bigvee_{d \in X}\left\{\left(\bigvee_{c \in X}(R(a, c) \wedge R(c, d)]\right) \wedge R(d, b)\right\}
$$$$
[\because R \text { is symmetric }]
$$$$
=\bigvee_{d \in X}[(R \circ R)(a, d) \wedge R(d, b)]
$$$$
\leq \bigvee_{d \in X}[R(a, d) \wedge R(d, b)][\because R \text { is transitive }]
$$$$
=(R \circ R)(a, b)
$$$$
\leq R(a, b) .[\because R \text { is transitive }]
$$

Thus $R_{\pi} \subset R$. On the other hand,

$$
R(a, b)
$$

$=R(a, a) \wedge R(b, b) \wedge R(a, b)$

$=\pi(a, R a) \wedge \pi(b, R b) \wedge E_{X / R}(R a, R b)$

[By the definitions of $\pi$ and $E_{X / R}$.]

$$
\leq \bigvee_{(c, d) \in X \times X}\left[\pi(a, R c) \wedge \pi(b, R d) \wedge E_{X / R}(R c, R d)\right]
$$

$=R_{\pi}(a, b) .\left[\right.$ By the definitions of $R_{\pi}$ ]

So $R \subset R_{\pi}$. Hence $R=R_{\pi}$.

Remark 5.1 Corollary 5.1 is the generalization of Theorem 3.22 in [6] in fuzzy setting.

Proposition 5.2 Let $f: X \rightarrow Y$ be a strong fuzzy mapping w.r.t. $I_{X} \in E(X)$ and $E_{Y} \in E(Y)$ and let ran $f=\{y \in Y: \exists x \in X$ such that $f(x, y)>0\} \subset Y$. Let $R$ be the fuzzy equivalence relation determined by $f$. We define two fuzzy relations $s$ and $t$ on $X / R \times \operatorname{ran} f$ and $\operatorname{ran} f \times Y$, respectively as follows: $s(R a, y)=f(a, y), \forall a \in X, \forall y \in \operatorname{ran} f$ and

$$
t\left(y, y^{\prime}\right)=\left\{\begin{array}{lll}
1 & \text { if } \quad y=y^{\prime} \\
0 & \text { if } \quad y \neq y^{\prime}, \forall y \in \operatorname{ran} f, \forall y^{\prime} \in Y .
\end{array}\right.
$$


Then $s$ is strong and bijective, $t$ is strong and injective and $f=t \circ s \circ \pi$.

Proof. (i) From Proposition 4.2, it is clear that $\pi: X \rightarrow X / R$ is a strong and strong surjective fuzzy mapping w.r.t. $I_{X}$ and $E_{X / R} \in E(X / R)$.

(ii) It is easily seen that $s: X / R \rightarrow \operatorname{ran} f$ is a fuzzy mapping w.r.t. $E_{X / R}$ and $E_{Y}$. Let $y \in \operatorname{ran} f$. Then $\exists x \in X$ such that $f(x, y)>0$. Thus $R x \in X / R$ and $s(R x, y)=f(x, y)>0$. So $\mathrm{s}$ is surjective. Now let $x_{1}, x_{2} \in X$ and $y_{1}, y_{2} \in \operatorname{ran} f$. Then

$$
\begin{aligned}
& E_{X / R}\left(R x_{1}, R x_{2}\right) \\
= & R\left(x_{1}, x_{2}\right) \\
= & \bigvee_{(c, d) \in Y \times Y}\left[f\left(x_{1}, c\right) \wedge f\left(x_{2}, d\right) \wedge E_{Y}(c, d)\right]
\end{aligned}
$$
by $f]$

$[\because R$ is the fuzzy equivalence relation determined

$$
\begin{aligned}
& \geq f\left(x_{1}, y_{1}\right) \wedge f\left(x_{2}, y_{2}\right) \wedge E_{Y}\left(y_{1}, y_{2}\right) \\
& =s\left(R x_{1}, y_{1}\right) \wedge s\left(R x_{2}, y_{2}\right) \wedge E_{Y}\left(y_{1}, y_{2}\right) .
\end{aligned}
$$

[By the definition of $s$.]

Thus $s$ is injective. Since $f$ is strong, it is clear that $s$ is strong. Hence $s$ is strong and bijective.

(iii) From the definition of $t$, it is clear that $t$ : ran $f \rightarrow Y$ is strong and injective fuzzy mapping w.r.t. $E_{Y}$ and $E_{Y}$.

(iv) Let $x \in X$ and let $y \in Y$. Then

$$
\begin{aligned}
& (t \circ s \circ \pi)(x, y) \\
= & {[(t \circ s) \circ \pi](x, y) } \\
= & \bigvee_{R a \in X / R}[\pi(x, R a) \wedge(t \circ s)(R a, y)] \\
= & \bigvee_{R a \in X / R}\left[R(a, x) \wedge\left(\bigvee_{z \in \operatorname{ran} f}[s(R a, z) \wedge t(z, y)]\right)\right]
\end{aligned}
$$

[By the definitions of $\pi$ and $t \circ s$ ]

$=\bigvee_{a \in X}\left[R(a, x) \wedge\left(\bigvee_{z \in \operatorname{ran} f}[f(a, z) \wedge t(z, y)]\right)\right]$

[By the definition of $s$.]

$=\bigvee_{z \in \operatorname{ran} f}[f(x, z) \wedge t(z, y)][\because R$ is reflexive $]$

$=f(x, y)$. [By the definition of $t]$

Thus $t \circ s \circ \pi=f$. This completes the proof.

The following is the immediate result of Propositions 3.6 and 5.2

Corollary 5.2 Let $f, s, t$ and $R$ be same as in Proposition 5.2. If $f$ is surjective [resp. strong surjective], then $t: \operatorname{ran} f \rightarrow Y$ is strong and bijective [resp. strong bijective] and hence $s: X / R \rightarrow Y$ is strong and bijective [resp. strong bijective].

Remark 5.2 Proposition 5.2 and Corollary 5.2 are the generalizations of Theorems 3.23 and 3.24 in [6] in fuzzy setting.
Proposition 5.3 Let $f: X \rightarrow Y$ be a strong fuzzy mapping w.r.t. $I_{X} \in E(X)$ and $E_{Y} \in E(Y)$. Let $R$ be the fuzzy equivalence relation on $X$ determined by $f$ and let $G$ be any fuzzy equivalence relation on $X$ such that $G \subset R$. We define the fuzzy relation $f / G$ on $X / G \times Y$ as follows:

$$
[f / G](G x, y)=f(x, y), \forall x \in X, \forall y \in Y .
$$

Then $f / G: X / G \rightarrow Y$ is a strong fuzzy mapping w.r.t. $E_{X / G} \in E(X / G)$ and $E_{Y}$. In this case, $f / G$ is called the fuzzy quatient of $f$ by $G$.

Proof. From the definition of $f / G$, it is clear that $f / G$ satisfies the condition (f.1). Let $G x_{1}, G x_{2} \in X / G$ and let $y_{1}, y_{2} \in Y$. Then

$$
\begin{aligned}
& (f / G)\left(G x_{1}, y_{1}\right) \wedge(f / G)\left(G x_{1}, y_{2}\right) \wedge \\
& E_{X / G}\left(G x_{1}, G x_{2}\right) \\
& =f\left(x_{1}, y_{1}\right) \wedge f\left(x_{2}, y_{2}\right) \wedge G\left(x_{1}, x_{2}\right) \\
& \leq f\left(x_{1}, y_{1}\right) \wedge f\left(x_{2}, y_{2}\right) \wedge R\left(x_{1}, x_{2}\right)[\text { Since } G \subset R] \\
& \quad=f\left(x_{1}, y_{1}\right) \wedge f\left(x_{2}, y_{2}\right) \wedge\left(\bigvee _ { ( c , d ) \in Y \times Y } \left[f\left(x_{1}, c\right) \wedge\right.\right. \\
& \left.\left.f\left(x_{2}, d\right) \wedge E_{Y}(c, d)\right]\right) .
\end{aligned}
$$$$
\text { by } f]
$$

$[\because R$ is the fuzzy equivalence relation determined

$=f\left(x_{1}, y_{1}\right) \wedge f\left(x_{2}, y_{2}\right) \wedge E_{Y}\left(c_{0}, d_{0}\right)$.

[Since $f$ is strong, $\exists c_{0}, d_{0} \in Y$ such that $\left.f\left(x_{1}, c_{0}\right)=f\left(x_{2}, d_{0}\right)=1\right]$

Since $f: X \rightarrow Y$ is fuzzy mapping w.r.t. $I_{X}$ and $E_{Y}$,

$f\left(x_{1}, y_{1}\right) \wedge f\left(x_{2}, y_{2}\right) \wedge I_{X}\left(x_{1}, x_{2}\right) \leq E_{Y}\left(y_{1}, y_{2}\right) .(5.2)$

By (5.1) and (5.2),

$$
\begin{aligned}
& f\left(x_{1}, y_{1}\right) \wedge f\left(x_{2}, y_{2}\right) \wedge I_{X}\left(x_{1}, x_{2}\right) \wedge E_{Y}\left(c_{0}, d_{0}\right) \\
\leq & E_{Y}\left(c_{0}, d_{0}\right) \wedge E_{Y}\left(y_{1}, y_{2}\right) \leq E_{Y}\left(y_{1}, y_{2}\right)
\end{aligned}
$$

Thus

$$
(f / G)\left(G x_{1}, y_{1}\right) \wedge(f / G)\left(G x_{2}, y_{2}\right) \wedge
$$

$E_{X / R}\left(G x_{1}, G x_{2}\right) \leq E_{Y}\left(y_{1}, y_{2}\right)$.

So $f / G$ satisfies the condition (f.2). Since $f$ is strong, it is clear that $f / G$ is strong. Hence $f / G: X / G \rightarrow Y$ is strong w.r.t. $E_{X / G}$ and $E_{Y}$.

Proposition 5.4 Let $f, R, G$ and $f / G$ be same as in Proposition 5.3. Then $R / G$ is the fuzzy equivalence relation on $X / G$ determined by $f / G$.

Proof. Let $R_{f / G}$ be the fuzzy equivalence relation on $X / G$ determined by $f / G$ and let $G a, G b \in X / G$. Then

$$
\begin{aligned}
& R_{f / G}(G a, G b) \\
= & \bigvee_{(c, d) \in Y \times Y}\left[(f / G)(G a, c) \wedge(f / G)(G b, d) \wedge E_{Y}(c, d)\right] \\
= & \bigvee_{(c, d) \in Y \times Y}\left[f(a, c) \wedge f(b, d) \wedge E_{Y}(c, d)\right] \\
= & R(a, b)[\text { By Proposition 5.1] } \\
= & R / G(G a, G b) .[\text { By Proposition 4.3] }
\end{aligned}
$$

Thus $R_{f / G}=R / G$. So $R / G$ is the fuzzy equivalence relation on $X / G$ determined by $f / G$. 
Remark 5.4 Proposition 5.4 is the generalization of Theorem 3.26 in $[6]$ in fuzzy setting.

Proposition 5.5 Let $R$ and $G$ be fuzzy equivalence relations on $X$ such that $G \subset R$. Then $\exists$ a strong and strong bijective fuzzy mapping $h:(X / G) /(R / G) \rightarrow$ $X / R$

Proof. By Proposition 4.2, $\exists$ a strong and strong surjective fuzzy mapping $\pi: X \rightarrow X / R$ w.r.t. $I_{X} \in E(X)$ and $E_{X / R} \in E(X / R)$. By Corollary 5.1 , it is clear that $R$ is the fuzzy equivalence relation on $X$ determined by $\pi$. Then, by Proposition 5.3, $\pi / G: X / G \rightarrow X / R$ is strong w.r.t. $E_{X / G} \in E(X / G)$ and $E_{X / R}$. Thus, by Proposition $5.4, R / G$ is the fuzzy equivalence relation determined by $\pi / G$. Since $\pi$ is strong surjective, $\pi / G$ is strong surjective. So, $\pi / G$ is strong and strong surjective. Hence, by Corollary $5.2, \exists$ a strong and strong bijective fuzzy mapping $h:(X / G) /(R / G) \rightarrow X / R$.

The following is the immediate result of Proposition 5.5.

Corollary 5.5 Let $R$ and $G$ be any fuzzy equivalence relations on $X$. Then :

(a) $\exists$ a bijective fuzzy mapping $g: X /(R \circ G) \rightarrow$ $(X / R) /(R \circ G / R)$.

(b) $\exists$ a bijective fuzzy mapping $h: X / R \rightarrow$ $(X / R \cap G) /(R / R \cap G)$.

Proposition 5.6 Let $f: X \rightarrow Y$ be a strong and strong surjective fuzzy mapping w.r.t. $I_{X} \in E(X)$ and $E_{Y} \in E(Y)$, and let $R$ be an fuzzy equivalence relation on $X$. Then $f^{2}(R)$ is a fuzzy equivalence relation on $Y$. In this case, $f^{2}(R)$ is called the image of $R$ under $f$.

Proof. Let $y \in Y$. Then

$$
\begin{aligned}
& f^{2}(R)(y, y) \\
= & \bigvee_{\left(x, x^{\prime}\right) \in X}\left[R\left(x, x^{\prime}\right) \wedge f^{2}\left(\left(x, x^{\prime}\right),(y, y)\right)\right] \\
= & \bigvee_{\left(x, x^{\prime}\right) \in X}\left[R\left(x, x^{\prime}\right) \wedge f(x, y) \wedge f\left(x^{\prime}, y\right)\right] \\
\geq & R\left(x_{0}, x_{0}\right)
\end{aligned}
$$

[Since $f$ is strong surjective, $\exists x_{0} \in X$ such that $\left.f\left(x_{0}, y\right)=1\right]$

$=1$.

Thus $f^{2}(R)$ is reflexive. From the definition of $f^{2}(R)$, it is clear that $f^{2}(R)$ is symmetric. Now let $y, y^{\prime \prime} \in Y$. Then

$$
\begin{aligned}
& {\left[f^{2}(R) \circ f^{2}(R)\right]\left(y, y^{\prime \prime}\right) } \\
= & \bigvee_{y^{\prime} \in Y}\left[f^{2}(R)\left(y, y^{\prime}\right) \wedge f^{2}(R)\left(y^{\prime}, y^{\prime \prime}\right)\right]
\end{aligned}
$$

$$
\begin{aligned}
& =\bigvee_{y^{\prime} \in Y}\left\{\left(\bigvee_{\left(x, x^{\prime}\right) \in X \times X}\left[R\left(x, x^{\prime}\right) \wedge f(x, y) \wedge f\left(x^{\prime}, y^{\prime}\right)\right]\right) \wedge\right. \\
& \left.\left(\bigvee_{\left(x^{\prime}, x^{\prime \prime}\right) \in X \times X}\left[R\left(x^{\prime}, x^{\prime \prime}\right) \wedge f\left(x^{\prime}, y^{\prime}\right) \wedge f\left(x^{\prime \prime}, y^{\prime \prime}\right)\right]\right)\right\} \\
& =\bigvee_{\left(x, x^{\prime \prime}\right) \in X \times X}\left[R\left(x, x_{0}\right) \wedge R\left(x_{0}, x^{\prime \prime}\right) \wedge f(x, y) \wedge\right.
\end{aligned}
$$$$
\left.f\left(x^{\prime \prime}, y^{\prime \prime}\right)\right]
$$

[Since $f$ is strong surjective, $\exists x_{0} \in X$ such that $\left.f\left(x_{0}, y^{\prime}\right)=1\right]$

$$
\begin{aligned}
& \leq \bigvee_{\left(x, x^{\prime \prime}\right) \in X \times X}\left[R\left(x, x^{\prime \prime}\right) \wedge f(x, y) \wedge f\left(x^{\prime \prime}, y^{\prime \prime}\right)\right] \\
& {[\because R \text { is transitive }]} \\
& =f^{2}(R)\left(y, y^{\prime \prime}\right)
\end{aligned}
$$

Thus $f^{2}(R) \circ f^{2}(R) \subset f^{2}(R)$. So $f^{2}(R)$ is transitive. Hence $f^{2}(R)$ is a fuzzy equivalence relation on $Y$.

Theorem 5.7 Let $f: X \rightarrow Y$ be strong and strong surjective w.r.t. $I_{X} \in E(X)$ and $E_{Y} \in E(Y)$, let $R$ be the fuzzy equivalence relation on $X$ induced by $f$ and let $G$ be any fuzzy equivalence relation on $Y$. Then :

(a) $R \subset f^{-2}(G)$.

(b) $H=f^{-2}(G)$ if and only it $G=f^{2}(H)$.

Hence $\exists$ a bijection $h: \operatorname{FER}(Y) \rightarrow \operatorname{FER}_{R}(X)$, where $\mathrm{FER}_{R}(X)$ denotes the set of all fuzzy equivalence relations on $X$ containing $R$.

Proof. (a) Let $x, x^{\prime} \in X$. Then

$$
=\bigvee_{\left(y, y^{\prime}\right) \in Y \times Y}^{R\left(x, x^{\prime}\right)}\left[f(x, y) \wedge f\left(x^{\prime}, y^{\prime}\right) \wedge E_{Y}\left(y, y^{\prime}\right)\right]
$$

[By Proposition 5.1]

$$
\begin{aligned}
\leq & \bigvee_{\left(y, y^{\prime}\right) \in Y \times Y}\left[f(x, y) \wedge f\left(x^{\prime}, y^{\prime}\right)\right] \\
= & \bigvee_{\left(y, y^{\prime}\right) \in Y \times Y}\left[G\left(y_{0}, y_{0}\right) \wedge f(x, y) \wedge f\left(x^{\prime}, y^{\prime}\right)\right] \\
& {\left[\because G\left(y_{0}, y_{0}\right)=1\right] } \\
= & \bigvee_{\left(y, y^{\prime}\right) \in Y \times Y}\left[G\left(y, y^{\prime}\right) \wedge f^{2}\left(\left(x, x^{\prime}\right),\left(y, y^{\prime}\right)\right)\right] \\
= & f^{-2}(G)\left(x, x^{\prime}\right) .
\end{aligned}
$$

Thus $R \subset f^{-2}(G)$. Then

(b) $(\Rightarrow)$ : Suppose $H=f^{-2}(G)$ and let $y, y^{\prime} \in Y$.

$$
\begin{aligned}
& f^{2}(H)\left(y, y^{\prime}\right) \\
= & \bigvee_{\left(x, x^{\prime}\right) \in X \times X}\left[H\left(x, x^{\prime}\right) \wedge f^{2}\left(\left(x, x^{\prime}\right),\left(y, y^{\prime}\right)\right)\right] \\
= & f_{\left(x, x^{\prime}\right) \in X \times X}\left[f^{-2}(G)\left(x, x_{0}, x_{0}^{\prime}\right) \wedge f(x, y) \wedge f\left(x^{\prime}, y^{\prime}\right)\right]
\end{aligned}
$$

[Since $f$ is strong surjective, $\exists x_{0}, x_{0}^{\prime} \in X$ such that $f\left(x_{0}, y\right)=f\left(x_{0}^{\prime}, y^{\prime}\right)=1$.]

$$
=\bigvee_{\left(z, z^{\prime}\right) \in Y \times Y}\left[G\left(z, z^{\prime}\right) \wedge f\left(x_{0}, z\right) \wedge f\left(x_{0}^{\prime}, z^{\prime}\right)\right]
$$


$=G\left(y, y^{\prime}\right) .\left[\because f\left(x_{0}, y\right)=f\left(x_{0}^{\prime}, y^{\prime}\right)=1\right]$

Thus $f^{2}(H)=G$.

$(\Leftarrow)$ : Suppose $f^{2}(H)=G$ and let $x, x^{\prime} \in X$. Then $f^{-2}(G)\left(x, x^{\prime}\right)$

$=\bigvee_{\left(y, y^{\prime}\right) \in Y \times Y}\left[G\left(y, y^{\prime}\right) \wedge f^{2}\left(\left(x, x^{\prime}\right),\left(y, y^{\prime}\right)\right)\right]$

$=\bigvee_{\left(y, y^{\prime}\right) \in Y \times Y}\left[f^{2}(H)\left(y, y^{\prime}\right) \wedge f(x, y) \wedge f\left(x^{\prime}, y^{\prime}\right)\right]$

$=f^{2}(H)\left(y_{0}, y_{0}^{\prime}\right)$

[Since $f$ is strong, $\exists y_{0}, y_{0}^{\prime} \in Y$ such that

$\left.f\left(x, y_{0}\right)=f\left(x^{\prime}, y_{0}^{\prime}\right)=1\right]$.

$=\bigvee\left[H(a, b) \wedge f\left(a, y_{0}\right) \wedge f\left(b, y_{0}^{\prime}\right)\right]$ $(a, b) \in X \times X$

$=H\left(x, x^{\prime}\right) .\left[\because f\left(x, y_{0}\right)=f\left(x^{\prime}, y_{0}^{\prime}\right)=1.\right]$

Thus $f^{-2}(G)=H$.

Now we define $h: \operatorname{FER}(Y) \rightarrow \operatorname{FER}_{R}(X)$ as follows: $\forall G \in \operatorname{FER}(Y), h(G)=f^{-2}(G)$. Then, by Proposition 5.6 and (a), clearly $h(G) \in \operatorname{FER}_{R}(X)$. It is easy to see that $h$ is bijective. This completes the proof.

\section{Acknowlegments}

By accepting some propositions of referees, we corrected some parts of this paper. Moreover, we used as examples (Example 3.6 and 3.9) two counterexamples proposed by them. So we would like to thank them for their advices.

\section{References}

[1] M.Demirci, "Fuzzy functions and their fundamental properties", Fuzzy Sets and Systems, vol.106, no. 2, pp. 239-246, 1999.

[2] J.A.Gouen, "L-fuzzy sets", J.Math.Anal.Appl., vol.18, no. 1, pp. 145-17, 1967.

[3] V.Murali, "Fuzzy equivalence relations", Fuzzy Sets and Systems, vol.30, pp. 155-163, 1989.
[4] W.C.Nemitz, "Fuzzy relations and fuzzy functions", Fuzzy Sets and Systems, vol.19, no. 8, pp. 177-191, 1986.

[5] S.V.Ovchinnikov, "Structure of fuzzy binary relations", Fuzzy Sets and Systems, vol.6, no. 2, pp. 169-195, 1981.

[6] C.C.Pinter, "Set Theory", Kyung Moon Publishers, 2002.

[7] P.M.Pu and Y.M.Liu, "Fuzzy topology II. Product and quotient spaces", J.Math.Anal.Appl. vol.77, no. 2, pp. 20-37, 1980.

[8] T.H.Yalvac, "Fuzzy sets and functions on fuzzy space", J.Math.Anal.Appl. vol.126, pp. 409-423, 1989.

[9] L.A.Zadeh, "Fuzzy sets", Inform. and Control, vol.8, pp. 338-353, 1965.

[10] L.A.Zadeh, "Similarity relations and fuzzy ordering", Inform. Soi, vol.3, pp. 117-200, 1971.

\section{Pyung Ki Lim}

Professor of Wonkwang University

Research Area: Fuzzy Topology, Dynamic Topology, etc.

E-mail : pklim@wonkwang.ac.kr

\section{Ga Hee Choi}

Research Area: Fuzzy Topology.

E-mail : ykgahee@naver.com

\section{Kul Hur}

Professor of Wonkwang University

Research Area: Fuzzy Topology, Fuzzy Algebra, etc.

E-mail : kulhur@wonkwang.ac.kr 